Y.-H. Chu, N.B. Suntzeff, J.E. Hesser, and D.A. Bohlender, eds.

\title{
HST/NICMOS Survey in the 30 Doradus Nebular Filaments
}

\author{
Rodolfo H. Barbá and Nolan R. Walborn \\ STScI, 3700 San Martin Dr., Baltimore, MD 21218, USA \\ Wolfgang Brandner \\ IPAC, Caltech, 770 South Wilson Ave., Pasadena, CA 91125, USA
}

Ronald G. Probst

CTIO, NOAO, Casilla 603, La Serena, Chile

Mónica Rubio

Depto. de Astronomía, Univ. de Chile, Casilla 36-D, Santiago, Chile

Eva K. Grebel

Lick Observatory, Univ. of California, Santa Cruz, CA 95064, USA

\begin{abstract}
On the basis of ample evidence accumulated during recent years for current star formation within the 30 Doradus Nebula, which appears to have been triggered by the massive central cluster (R136) in and just beyond the curved nebular filaments surrounding it, an HST/NICMOS survey of these regions was undertaken.
\end{abstract}

In contrast with prior ideas that the 30 Doradus Nebula is essentially an evolved $\mathrm{H}$ II region, during recent years observational evidence of the birth of a new generation of massive stars has grown. Actually, nebular filaments encircling the core can now be recognized as very active star-forming regions, where winds and radiation from massive stars in the R136 core are interacting with surrounding molecular clouds, triggering a new stellar generation. Among that observational evidence, groundbased observations show many IR sources distributed in or beyond (with respect to R136) the nebular filaments (Rubio et al. 1998 and references therein); early O stars embedded in dense nebular knots (Walborn \& Blades 1997) are spatially associated with those IR sources (Rubio et al. 1998); and WFPC2 images reveal numerous nebular microstructures, such as bright and dark dusty globules and pillars (Scowen et al. 1998; Rubio et al. 1998), symptoms of active star formation. Also, improved CO (Johansson et al. 1998) and $\mathrm{H}_{2}$ (Probst \& Rubio 1998) observations clearly demonstrate huge molecular concentrations beyond the west and northeast filaments.

On the basis of this observational evidence, HST/NICMOS observations were carried out in selected fields on the nebular filaments, under HST GO Program 7819, during February and March 1998. NIC2 broadband images in 

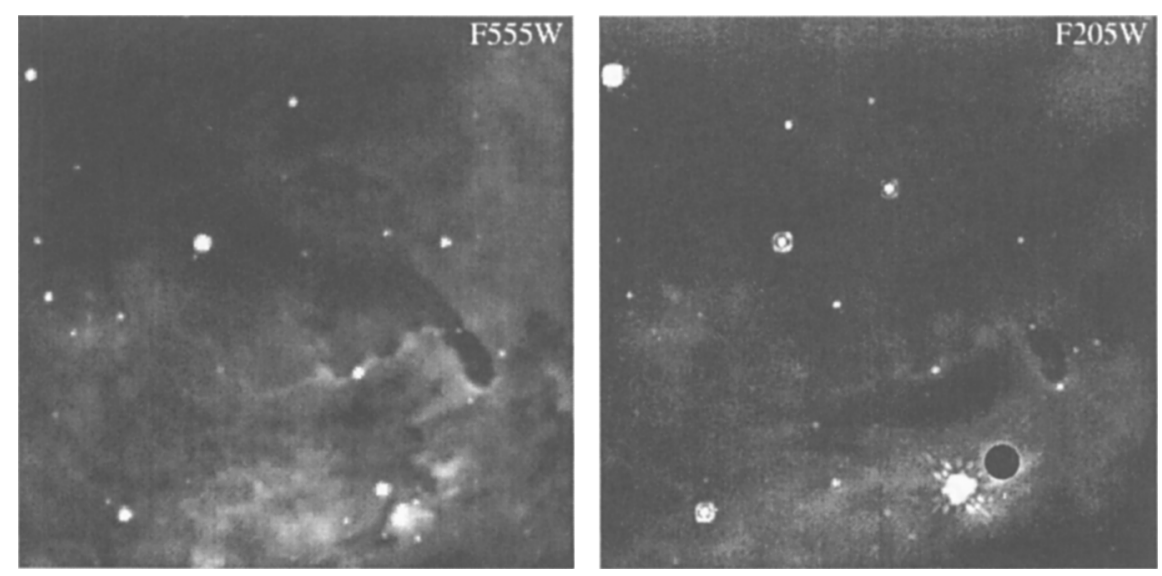

Figure 1. An example of WFPC2/F555W (left) and NIC2/F205W (right) images of filaments in the 30 Doradus Nebula.

F110W, F160W, and F205W filters were obtained in 15 fields. NIC2 narrowband images in F212N $\left(\mathrm{H}_{2}\right), \mathrm{F} 215 \mathrm{~N}$ (continuum), and $\mathrm{F} 216 \mathrm{~N}(\mathrm{Br} \gamma)$ were obtained in three selected fields, two in common with broadband ones. In addition, the NIC1 and NIC3 cameras were operated in parallel with the NIC2 camera. In NIC1 the F160W filter was used, and in NIC3 F187N ( $\mathrm{Pa} \alpha$ ), F190N (continuum), in addition to the same narrowband set as with NIC2. Further information about these observations is presented in these proceedings by Walborn \& Barbá. A detailed morphological description of several NICMOS fields and their implications for the new view of 30 Doradus has been published (Walborn et al. 1999).

As an example, Fig. 1 shows a comparison between a WFPC2/F555W (left) and NIC2/F205W (right) image of a dark cloud shaped like a hand and "Finger of God"; it is located in a loop on the NE filament and points directly toward R136. In the NICMOS image it is possible to see several IR sources by direct comparison with the WFPC2 image. NICMOS reveals that the structure is reminiscent of the "Finger of ET", with an IR star exactly at its tip. Each image is $19{ }^{\prime \prime} 2$ on a side. The dark spot in the NIC2 image corresponds to the coronographic hole.

Acknowledgments. Support for this work was provided by NASA through grants numbers GO-7819.01-96A and AR-7545.01-96A from STScI, which is operated by AURA, Inc., under NASA contract NAS 5-26555.

\section{References}

Johansson, L.E.B., et al. 1998, 331, 857

Rubio, M., et al. 1998, AJ, 116, 1708

Scowen, P.A., et al. 1998, AJ, 116, 163

Walborn, N.R., et al. 1999, AJ, 117, 225

Walborn, N.R., \& Blades, J.C. 1997, ApJS, 112, 457 